\title{
Effect of High Glucose on Stress-Induced Senescence of Nucleus Pulposus Cells of Adult Rats
}

\author{
Jae-Gwan Kong, Jong-Beom Park, Donghwan Lee, Eun-Young Park \\ Department of Orthopedic Surgery, Uijeongbu St. Mary's Hospital, The Catholic University of Korea College of Medicine, Uijeongbu, Korea
}

\section{Study Design: In vitro cell culture model.}

Purpose: We investigated the effect of diabetes mellitus (DM) on senescence of adult nucleus pulposus (NP) cells.

Overview of Literature: DM is a major public health issue worldwide, especially adult-onset (type 2) DM. DM is also thought to be an important etiological factor in disc degeneration. Hyperglycemia is considered to be a major causative factor in the development of DM-associated diseases through senescence. However, little is known about the effects of DM on senescence in adult NP cells.

Methods: Adult NP cells were isolated from 24-week-old rats, cultured, and placed in either 10\% fetal bovine serum (FBS, normal control) and $10 \%$ FBS plus two different high glucose concentrations (0.1 M or $0.2 \mathrm{M}$; experimental conditions) for 1 or 3 days. We identified and quantified the occurrence of senescence in adult rat NP cells using senescence-associated-beta-galactosidase (SA- $\beta$-Gal) staining. We also investigated the expression of proteins related to the replicative senescence (p53-p21-pRB) and stress-induced premature senescence (p16-pRB) pathways.

Results: The mean SA- $\beta$-Gal-positive percentage was increased in adult rat NP cells treated with high glucose in a dose- and timedependent manner. Both high glucose levels increased the expression of p16 and pRB proteins in adult rat NP cells. However, the levels of p53 and p21 proteins were decreased in adult rat NP cells treated with both high glucose concentrations.

Conclusions: The current study demonstrated that high glucose accelerated stress-induced senescence in adult rat NP cells in a dose- and time-dependent manner. Accelerated stress-induced senescence in adult NP cells could be an emerging risk factor for intervertebral disc degeneration in older patients with DM. These results suggest that strict blood glucose control is important in prevent or delaying intervertebral disc degeneration in older patients with DM.

Keywords: Senescence; Adult nucleus pulposus cells; High glucose; Intervertebral disc degeneration

\section{Introduction}

Cellular senescence is an irreversible growth arrest state that can occur after repeated cell division (replicative senescence) and also can be induced prematurely by stress conditions such as oxidative stress, DNA damage, and mitogenic stress (stress-induced senescence) [1-3]. Senescent cells cannot divide even if stimulated by mitogens, but they are not physiologically inert and display altered phenotypic changes and gene expression patterns. They also secrete degradative enzymes, inflammatory cytokines, and growth factors, which lead to a shift from

Received Mar 12, 2014; Revised Apr 17, 2014; Accepted Apr 17, 2014

Corresponding author: Jong-Beom Park

Department of Orthopedic Surgery, Uijeongbu St. Mary's Hospital, The Catholic University of Korea College of Medicine,

271 Cheonbo-ro, Uijeongbu 480-717, Korea

Tel: +82-31-820-3578, Fax:+82-31-847-3671, E-mail: spinepjb@catholic.ac.kr 
extracellular matrix synthesis to degradation. Thus, senescence is considered to be a major factor contributing to age- or disease-associated tissue dysfunction and pathology. Replicative senescence is mediated by the p53p21-pRB pathway whereas stress-induced senescence involves the p16-pRB pathway [4-6]. Senescent cells are characterized by telomere shortening and decreased telomerase activity. Additionally, senescence-associatedbeta-galactosidase (SA- $\beta$-Gal) activity is used to identify the occurrence of senescence [7-9].

Diabetes mellitus (DM) is a major public health problem worldwide. There are two main types of DM. Type $1 \mathrm{DM}$ results from the body's failure to produce insulin and is referred to as "insulin-dependent DM (IDDM)" or "juvenile-onset DM." Type 2 DM results from insulin resistance and is referred to as "non-insulin-dependent DM (NIDDM)" or "adult-onset DM." Approximately $90 \%$ of all cases of DM are type $2 \mathrm{DM}$. The number of DM patients will reach 300 million in 2025 (International Diabetes Federation, 2001) [10]. DM prevalence increases with age, and the number of older persons with DM is expected to grow simply as the elderly population increases in number. Untreated DM can cause many complications, such as cardiovascular disease, chronic renal failure, retinopathy, and neuropathy [11].

DM is also considered to be an important etiological factor in intervertebral disc degeneration [12-14]. Previous studies have reported a higher incidence of degenerative disc diseases in patients with DM than in the non-DM population [15]. A considerable proportion of the patients who undergo spine surgery are DM patients and their surgical outcomes have been reported to be poor versus non-DM patients [16,17]. Recently, a few studies have reported association between senescence and intervertebral disc degeneration [18-23]. However, the senescence of adult nucleus pulposus (NP) cells as a biological mechanism, as well as the role that senescence plays in intervertebral disc degeneration, is not well understood.

The intervertebral disc is a highly specialized structure that consists of an outer annulus fibrosus (AF), which surrounds the inner NP. The NP undergoes a chronological transition from a notochordal to a fibrocartilaginous NP. This transition accompanies changes in cell types, from notochordal cells to so-called chondrocytelike cells. In humans, this transition is usually completed by the second decade [24]. Additionally, there are some differences in the composition of extracellular matrix produced by notochordal cells and adult NP chondrocyte-like cells. Glucose-mediated increases in oxidative stress, such as reactive oxygen species (ROS), is a major biochemical pathway of DM-associated disease [2527]. The main source of endogenous ROS is the mitochondria in most mammalian cell types [28]. ROS are thought to play roles in various cellular processes, such as senescence, which appear to cause cellular damage and lack a physiological function. Thus, accumulation of ROS has been reported to be associated with a variety of diseases including neurodegenerative diseases, DM, cancer, premature aging, and inflammatory disorders $[29,30]$. It is important to clarify the effects of DM on senescence of adult NP cells so as to prevent or to delay intervertebral disc degeneration in older patients with DM. Thus, in the current study, we investigated the effects of DM on senescence of adult NP cells and intervertebral disc degeneration.

\section{Materials and Methods}

\section{Adult rat NP cells: culture and high glucose treatment}

All lumbar intervertebral discs (L1-6) were harvested from 24-week-old male Sprague Dawley rats (Orient Bio., Seoul, Korea) immediately after they were sacrificed. The discs were dissected carefully under a microscope to obtain only the NP tissues, and the harvested NP tissues were pooled in $\alpha$-minimum essential medium ( $\alpha$-MEM; Gibco BRL, Grand Island, NY, USA). The cells were released from the NP tissues in Han's Balanced Salt Solution (Hyclone, Ottawa, ON, Canada) with $0.02 \%$ pronase (Sigma-Aldrich, St. Louis, MO, USA) with vigorous pipetting. The cells were cultured in a complete medium: a-MEM supplemented with $10 \%$ fetal bovine serum (FBS, Hyclone), and $1 \%$ penicillin-streptomycin (Gibco BRL) at $37^{\circ} \mathrm{C}$ in a humidified atmosphere (95\% air, $\left.5 \% \mathrm{CO}_{2}\right)$. After the cells grew to confluence, they were split once (passage 1 ) and grown to confluence again. The cells were then trypsinized, washed, and plated on 6- or 12-well culture plates. When the cells reached $80 \%-90 \%$ confluence, the cells were placed in FBS (normal control) of $10 \%$ FBS plus two different high glucose concentrations $(0.1 \mathrm{M}$ or $0.2 \mathrm{M}$, experimental condition) for 1 or 3 days. This study was approved by the Institution's Animal Care and Use Committee. 


\section{Senescence of adult rat NP cells}

SA- $\beta$-Gal activity in the adult rat NP cells was determined using a SA- $\beta$-Gal staining kit (Cell Signaling Technology). The cells were plated on poly (L-lysine)-coated 12 -mm glass cover slips and fixed with $2 \%$ formaldehyde and $0.2 \%$ glutaraldehyde in phosphate-buffered saline (PBS) for 15 minutes at room temperature. The slides were rinsed with PBS and then incubated for 8 hours at $37^{\circ} \mathrm{C}$ with fresh SA- $\beta$-gal staining solution containing $40 \mathrm{mM}$ citric acid/sodium phosphate $(\mathrm{pH} 6.0), 150 \mathrm{mM}$ $\mathrm{NaCl}, 2 \mathrm{mM} \mathrm{MgCl}$, $5 \mathrm{mM}$ potassium ferrocyanide, 5 $\mathrm{mM}$ potassium ferricyanide, and $1 \mathrm{mg} / \mathrm{mL}$ of 5-bromo4-chloro-3-indolyl- $\beta$-D-galactopyranoside (X-gal). After rinsing with $70 \%$ glycerol, blue-stained cells were identified as senescent cells by microscopy. Total adult NP and AF cells and SA- $\beta$-Gal-positive adult rat NP cells on the whole section were counted $(\times 200)$, and the percentage of SA- $\beta$-Gal-positive adult rat NP cells was calculated.

\section{Expression of $\mathrm{p} 53, \mathrm{p} 21, \mathrm{pRB}$, and $\mathrm{p} 16$}

Adult rat NP cells were plated on poly (L-lysine)-coated 12 -mm glass cover slips. The cells were fixed with $4 \%$ paraformaldehyde in PBS for 15 minutes at room temperature and washed with PBS, pH 7.4. The cells were permeabilized with $0.2 \%$ TritonX-100 in PBS for 10 minutes. After incubation for 30 minutes at room temperature with PBS containing 3\% BSA (blocking solution), cells were incubated overnight at $4^{\circ} \mathrm{C}$ with the primary antibody (1:100 dilution) for p53 (Novus Biologicals, CO, USA), p21 (Abcam Plc, Cambridge, UK), pRB (Biorbyt Ltd., Cambridge, UK), or p16 (Santa Cruz Biotechnology, Santa Cruz, CA, USA). After washes, the anti-rabbit Alexa Fluor 488 (Invitrogen, Carlsbad, CA, USA) and antimouse Alexa Fluor 555 (Invitrogen) fluorescent conjugated IgG antibodies (1:5,000 dilution) in blocking solution were added and incubated for 1 hour at room temperature in the dark. The cover slips were mounted on object glasses with mounting medium with 4,6-diamidino-2phenylindole (VECTOR, Burlingame, CA, USA). Fluorescent signals were detected by fluorescence microscopy (Olympus Co., Tokyo, Japan).

\section{Statistical analyses}

All experiments were conducted independently three times, and the results are expressed as means and standard deviations of the three tests. Statistical analyses were made using the paired-samples $t$ test. A $p$-values $<0.05$ were considered to indicate statistical significance.

\section{Results}

\section{Increased senescence of adult rat NP cells}

The mean SA- $\beta$-Gal-positive percentage was increased significantly in adult NP cells treated with two high glucose concentrations for 1 day or 3 days in a dose- and time-dependent manner when compared with the control (Fig. 1).

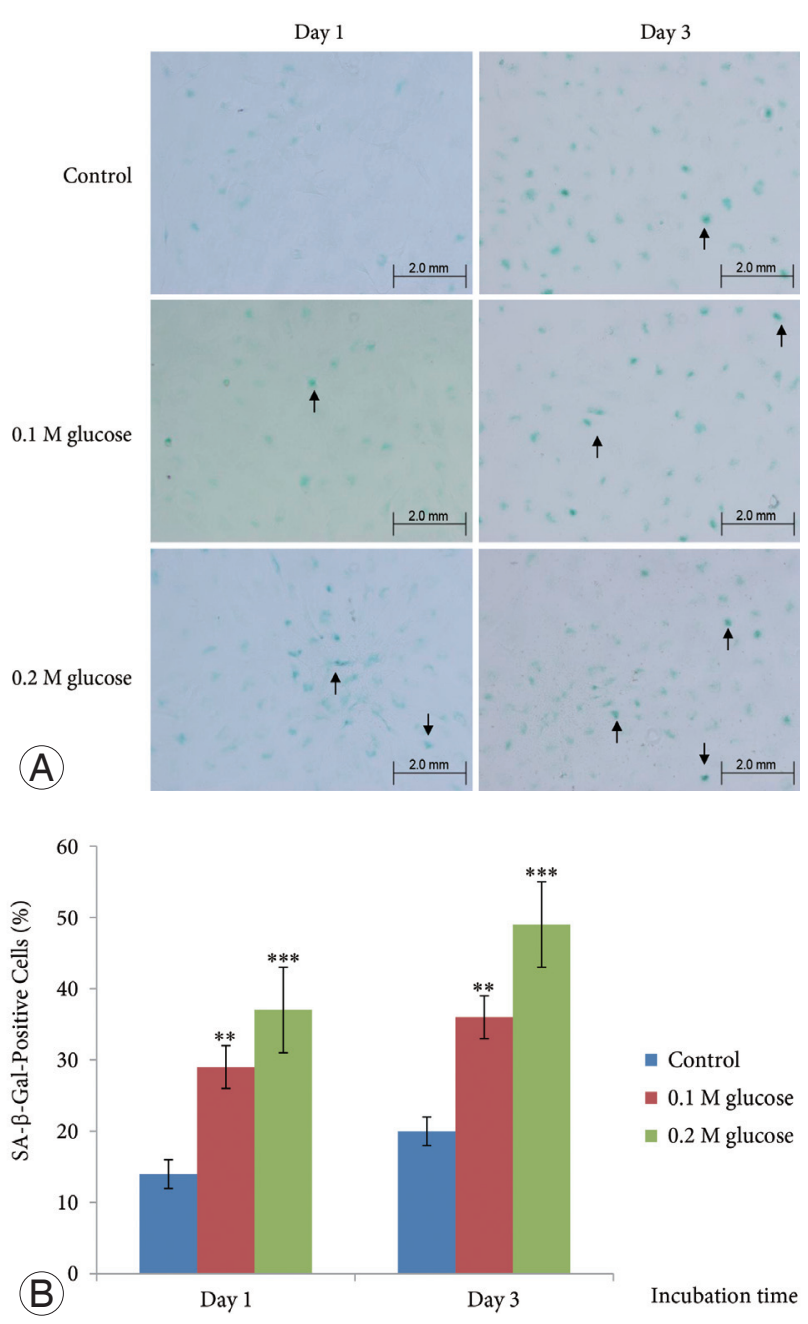

Fig. 1. The mean SA- $\beta$-gal-positive percentage (arrows) was increased in adult nucleus pulposus cells treated with two high glucose concentrations for 1 or 3 days in dose- and time-dependent manner, when compared with the control $(\mathbf{A}, \mathbf{B}) .{ }^{* * *} p<0.001$; ${ }^{* *} p<0.01$. 


\section{Stress-induced senescence of adult rat NP cells}

Immunofluorescence demonstrated that the two high glucose concentrations enhanced the expression of p16 protein (red color; Fig. 2) in nuclei of adult rat NP cells for

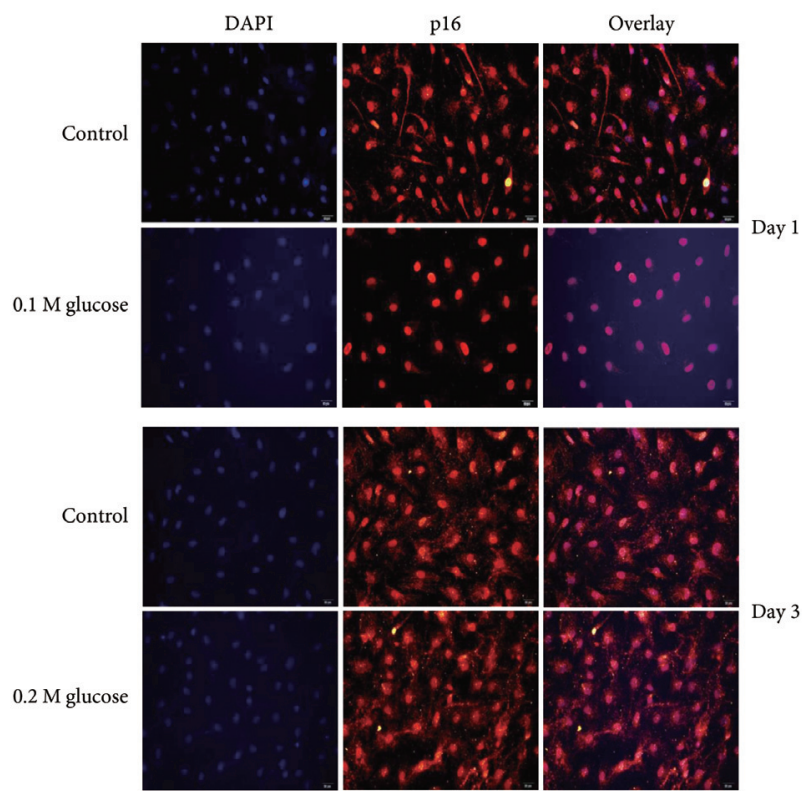

Fig. 2. Immunofluorescence demonstrated enhanced expression of p16 (red color) protein due to two high glucose concentrations in adult nucleus pulposus cells for 1 or 3 days, when compared with the control.

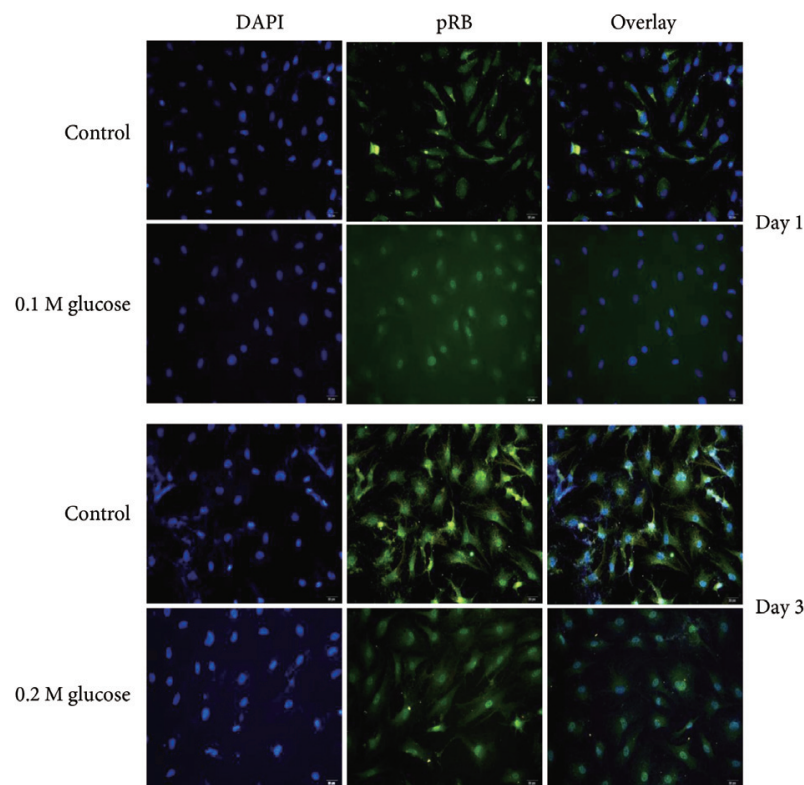

Fig. 3. Immunofluorescence demonstrated enhanced expression of pRB (green color) protein due to two high glucose concentrations in adult nucleus pulposus cells for 1 or 3 days, when compared with the control.
1 and 3 days when compared with the control. Additionally, immunofluorescence demonstrated that the two high glucose concentrations enhanced the expressions of $\mathrm{pRB}$ protein (green color; Fig. 3 ) in the cytoplasm as well as in nuclei of adult rat NP cells for 1 and 3 days, whereas the expression of $\mathrm{pRB}$ (green color) potential was observed only in the nuclei of adult rat NP cells in the normal control. Immunofluorescence demonstrated that the two high glucose concentrations decreased the expression of p53 protein (green color; Fig. 4) in adult rat NP cells for 1 and 3 days, when compared with the control. Additionally, the expression of p21 protein (green color; Fig. 5) was decrease in adult rat NP cells treated with the two high glucose concentrations, when compared with the normal control.

\section{Discussion}

The current findings demonstrate that two high glucose concentrations significantly increased the occurrence of senescence in adult rat NP cells in a dose- and timedependent manner. The expression of proteins related to the stress-induced senescence (p16-pRB) pathway was enhanced in adult rat NP cells treated with both high glucose concentrations. However, the expression of proteins related to replicative senescence (p53-p21) were

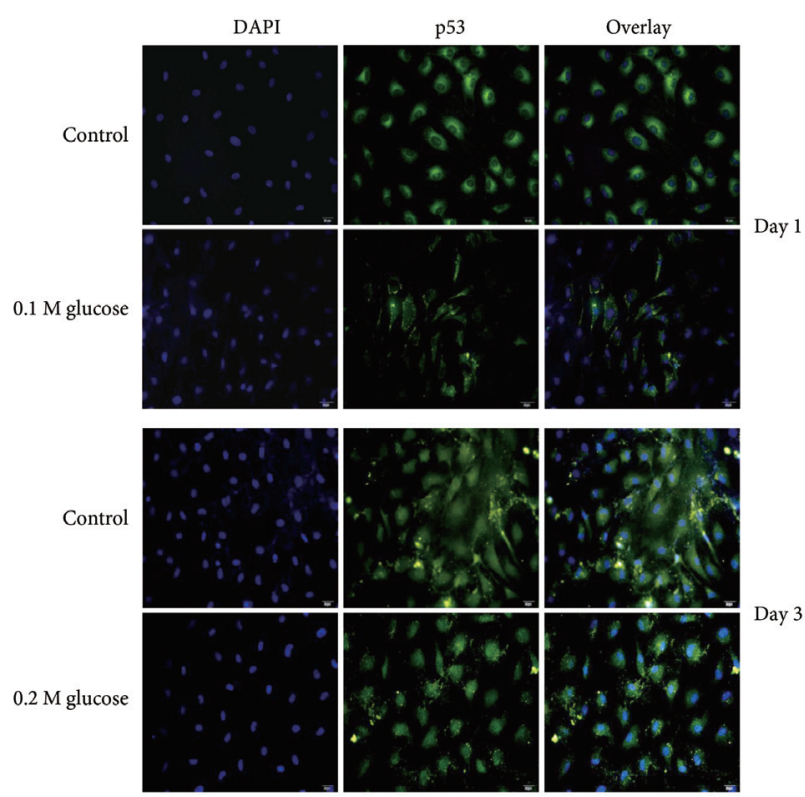

Fig. 4. Immunofluorescence demonstrated decreased expression of p53 (green color) protein due to two high glucose concentrations in adult nucleus pulposus cells for 1 or 3 days, when compared with the control. 


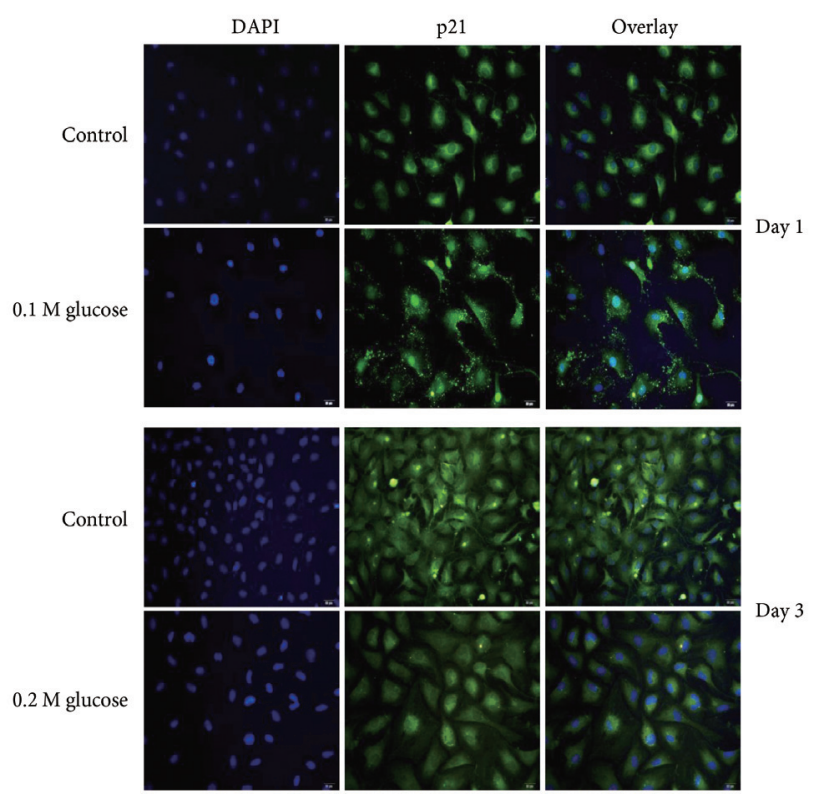

Fig. 5. Immunofluorescence decreased expression of p21 (green color) protein due to two high glucose concentrations in adult nucleus pulposus cells for 1 or 3 days when compared with the control.

decreased in adult rat NP cells treated with both high glucose concentrations. These results suggest high glucose accelerates stress-induced senescence in adult rat NP cells rather than replicative senescence, in a dose- and timedependent manner. Thus, accelerated stress-induced senescence of adult NP cells could be an emerging risk factor for intervertebral disc degeneration in older patients with DM. These results suggest that strict blood glucose control is important in preventing or delaying intervertebral disc degeneration in older patients with DM.

Senescence may have evolved as a mechanism to prevent cells with damaged DNA from replicating, thus preventing tumor formation [31,32]. Replicative senescence is associated with changes in DNA structure and function including telomere shortening and decreased telomerase activity [1-3]. However, senescence appears to be more complex than simple cell-cycle arrest occurring after a finite number of cell divisions. Progressive telomere shorting due to repeated cycles of cell division does not explain senescence in post-mitotic cells, such as neurons, or quiescent cells, such as chondrocytes. Stress-induced senescence can occur from diverse stimuli including ultraviolet radiation, oxidative damage, activated oncogenes, and chronic inflammation [4-6]. Oxidative damage to DNA can directly contribute to stress-induced senescence and, because the ends of chromosomes are particularly sensitive to oxidative damage, can result in telomere shortening similar to that seen with replicative senescence. Oxidative stress has been found to induce cell senescence in vitro and there is in vivo evidence for age-related oxidative stress in many tissues [4-6]. As additional evidence for a role of ROS in aging, increased expression of the antioxidant enzyme catalase in mitochondria of transgenic mice can extend life-span and reduce age-related changes in tissues, such as the heart [1-3]. It is known that mitochondria, when damaged, release harmful ROS into the cytosol [25-30]. A hallmark of senescence is the expression of SA- $\beta$-Gal; however, it is not strictly specific for senescent cells, because non-senescent cells that have a high lysosomal content also stain positive in this assay [7-9].

Before this study, it had not been confirmed whether DM caused senescence in adult NP cells. The current study showed a higher percentage of SA- $\beta$-Gal-positive staining, meaning a higher rate of the occurrence of senescence, in adult rat NP cells treated with high glucose concentrations compared with the normal control. To supplement shortcomings of SA- $\beta$-Gal staining, we also performed immunofluorescence staining to determine the expression of proteins, such as p53, p21, pRB, and $\mathrm{p} 16-\mathrm{pRB}$, related to the replicative and stress-induced senescence pathways. Immunofluorescence showed that senescent adult rat NP cells undergoing high glucose treatment significantly expressed $\mathrm{p} 16$ and $\mathrm{pRB}$ proteins, whereas the expression levels of p53 and p21 proteins were decreased versus the normal control. These results indicate that high glucose condition accelerates stress-induced senescence (p16-pRB) predominantly in adult rat NP cells, rather than replicative senescence. Additionally, our study demonstrated that the percentage of senescence of adult rat NP cells increased in proportion to duration of high glucose treatment. This may explain the higher incidence of intervertebral disc degeneration in patients with a long duration of DM, which results in a higher incidence of degenerative disc diseases.

There are some limitations to the current study. First, we did not investigate telomere length or telomerase activity. Telomere shortening is a characteristic finding for cellular senescence. Second, we did not investigate the excessive generation of oxidative stress, such as ROS, through mitochondrial damage due to the high glucose conditions. However, considering the current results and 
previous studies, we suggest that excessive generation of oxidative stress under high glucose conditions accelerates stress-induced senescence in adult rat NP cells. Further studies are needed to confirm this. Third, this in vitro high glucose-induced diabetic model does not perfectly reflect in vivo aspects of intervertebral disc degeneration with DM, especially in terms of high glucose concentrations and culture period. Thus, further in vivo studies are also needed.

\section{Conclusions}

The current findings demonstrate that high glucose conditions accelerated stress-induced senescence of adult rat NP cells in a time-dependent manner. This results in dysfunction of adult rat NP cells, resulting in intervertebral disc degeneration. Thus, accelerated stress-induced senescence of adult NP cells could be an emerging risk factor for disc degeneration in older patients with DM. Thus, these results suggest that strict blood glucose control is important in preventing or delaying intervertebral disc degeneration in older patients with DM.

\section{Conflict of Interest}

No potential conflict of interest relevant to this article was reported.

\section{References}

1. Tsirpanlis G. Cellular senescence, cardiovascular risk, and CKD: a review of established and hypothetical interconnections. Am J Kidney Dis 2008;51:131-44.

2. Shimada H, Sakakima H, Tsuchimochi K, et al. Senescence of chondrocytes in aging articular cartilage: GADD45beta mediates p21 expression in association with C/EBPbeta in senescence-accelerated mice. Pathol Res Pract 2011;207:225-31.

3. Unterluggauer H, Hampel B, Zwerschke W, JansenDurr P. Senescence-associated cell death of human endothelial cells: the role of oxidative stress. Exp Gerontol 2003;38:1149-60.

4. Khan IM, Gilbert SJ, Caterson B, Sandell LJ, Archer CW. Oxidative stress induces expression of osteoarthritis markers procollagen IIA and 3B3(-) in adult bovine articular cartilage. Osteoarthritis Cartilage 2008;16:698-707.
5. Chainiaux F, Magalhaes JP, Eliaers F, Remacle J, Toussaint O. UVB-induced premature senescence of human diploid skin fibroblasts. Int J Biochem Cell Biol 2002;34:1331-9.

6. Gorgoulis VG, Pratsinis H, Zacharatos P, et al. p53dependent ICAM-1 overexpression in senescent human cells identified in atherosclerotic lesions. Lab Invest 2005;85:502-11.

7. Severino J, Allen RG, Balin S, Balin A, Cristofalo VJ. Is beta-galactosidase staining a marker of senescence in vitro and in vivo? Exp Cell Res 2000;257:162-71.

8. Dimri GP, Lee X, Basile G, et al. A biomarker that identifies senescent human cells in culture and in aging skin in vivo. Proc Natl Acad Sci U S A 1995; 92:9363-7.

9. Kurz DJ, Decary S, Hong Y, Erusalimsky JD. Senescence-associated (beta)-galactosidase reflects an increase in lysosomal mass during replicative ageing of human endothelial cells. J Cell Sci 2000;113 (Pt 20):3613-22.

10. Alberti KG, Zimmet P, Shaw J. Metabolic syndrome: a new world-wide definition. A Consensus Statement from the International Diabetes Federation. Diabet Med 2006;23:469-80.

11. McClelland AD, Kantharidis P. microRNA in the development of diabetic complications. Clin Sci (Lond) 2014;126:95-110.

12. Won HY, Park JB, Park EY, Riew KD. Effect of hyperglycemia on apoptosis of notochordal cells and intervertebral disc degeneration in diabetic rats. J Neurosurg Spine 2009;11:741-8.

13. Park EY, Park JB. Dose- and time-dependent effect of high glucose concentration on viability of notochordal cells and expression of matrix degrading and fibrotic enzymes. Int Orthop 2013;37:1179-86.

14. Park EY, Park JB. High glucose-induced oxidative stress promotes autophagy through mitochondrial damage in rat notochordal cells. Int Orthop 2013;37: 2507-14.

15. Mobbs RJ, Newcombe RL, Chandran KN. Lumbar discectomy and the diabetic patient: incidence and outcome. J Clin Neurosci 2001;8:10-3.

16. Sakellaridis N. The influence of diabetes mellitus on lumbar intervertebral disk herniation. Surg Neurol 2006;66:152-4.

17. Simpson JM, Silveri CP, Balderston RA, Simeone FA, An HS. The results of operations on the lumbar spine 
in patients who have diabetes mellitus. J Bone Joint Surg Am 1993;75:1823-9.

18. Zhao CQ, Wang LM, Jiang LS, Dai LY. The cell biology of intervertebral disc aging and degeneration. Ageing Res Rev 2007;6:247-61.

19. Gruber HE, Watts JA, Hoelscher GL, et al. Mitochondrial gene expression in the human annulus: in vivo data from annulus cells and selectively harvested senescent annulus cells. Spine J 2011;11:782-91.

20. Roberts S, Evans EH, Kletsas D, Jaffray DC, Eisenstein SM. Senescence in human intervertebral discs. Eur Spine J 2006;15 Suppl 3:S312-6.

21. Gruber HE, Ingram JA, Norton HJ, Hanley EN Jr. Senescence in cells of the aging and degenerating intervertebral disc: immunolocalization of senescenceassociated beta-galactosidase in human and sand rat discs. Spine (Phila Pa 1976) 2007;32:321-7.

22. Kletsas D. Senescent cells in the intervertebral disc: numbers and mechanisms. Spine J 2009;9:677-8.

23. Kim KW, Chung HN, Ha KY, Lee JS, Kim YY. Senescence mechanisms of nucleus pulposus chondrocytes in human intervertebral discs. Spine J 2009;9:658-66.

24. Kim KW, Lim TH, Kim JG, Jeong ST, Masuda K, An HS. The origin of chondrocytes in the nucleus pulposus and histologic findings associated with the transition of a notochordal nucleus pulposus to a fibrocartilaginous nucleus pulposus in intact rabbit in- tervertebral discs. Spine (Phila Pa 1976) 2003;28:98290.

25. Baynes JW. Role of oxidative stress in development of complications in diabetes. Diabetes 1991;40:405-12.

26. Nishikawa T, Araki E. Impact of mitochondrial ROS production in the pathogenesis of diabetes mellitus and its complications. Antioxid Redox Signal 2007;9: 343-53.

27. Dorn GW 2nd. Mechanisms of non-apoptotic programmed cell death in diabetes and heart failure. Cell Cycle 2010;9:3442-8.

28. Victor VM, Rocha M, Herance R, Hernandez-Mijares A. Oxidative stress and mitochondrial dysfunction in type 2 diabetes. Curr Pharm Des 2011;17:3947-58.

29. Brandl A, Hartmann A, Bechmann V, Graf B, Nerlich $\mathrm{M}$, Angele P. Oxidative stress induces senescence in chondrocytes. J Orthop Res 2011;29:1114-20.

30. Furukawa A, Tada-Oikawa S, Kawanishi S, Oikawa S. $\mathrm{H} 2 \mathrm{O} 2$ accelerates cellular senescence by accumulation of acetylated p53 via decrease in the function of SIRT1 by NAD+ depletion. Cell Physiol Biochem 2007;20:45-54.

31. Ben-Porath I, Weinberg RA. The signals and pathways activating cellular senescence. Int J Biochem Cell Biol 2005;37:961-76.

32. Campisi J. Cellular senescence as a tumor-suppressor mechanism. Trends Cell Biol 2001;11:S27-31. 